DRAFT VERSION JUNE 20, 2017

Typeset using $\mathrm{IAT}_{\mathrm{E}} \mathrm{X}$ manuscript style in AASTeX61

\title{
AN UPDATED 2017 ASTROMETRIC SOLUTION FOR BETELGEUSE
}

\author{
G. M. Harper,${ }^{1}$ A. Brown,${ }^{1}$ E. F. Guinan, ${ }^{2}$ E. O'Gorman,${ }^{3}$ A. M. S. Richards,${ }^{4}$ \\ P. Kervella, ${ }^{5,6}$ AND L. Decin ${ }^{7}$
}

\footnotetext{
${ }^{1}$ Center for Astrophysics and Space Astronomy, University of Colorado, Boulder, CO 80309, USA

${ }^{2}$ Villanova University, PA 19085, USA

${ }^{3}$ Dublin Institute for Advanced Studies, Dublin 2, Ireland

${ }^{4}$ Jodrell Bank Centre for Astrophysics, School of Physics and Astronomy, University of Manchester, UK

${ }^{5}$ Unidad Mixta Internacional Franco-Chilena de Astronomía (UMI 3386), Departamento de Astronomía, Universidad de Chile, Santiago, Chile.

${ }^{6}$ Observatoire de Paris, PSL Research University, CNRS, UPMC, Univ. Paris-Diderot, France.

${ }^{7}$ Instituut voor Sterrenkunde, Katholieke Universiteit Leuven, Celestijnenlaan 200D, 3001 Leuven, Belgium
}

(Received receipt date; Revised revision date; Accepted acceptance date; Published published date)

Submitted to AJ

\begin{abstract}
We provide an update for the astrometric solution for the Type II supernova progenitor Betelgeuse using the revised Hipparcos Intermediate Astrometric Data (HIAD) of van Leeuwen, combined with existing VLA and new e-MERLIN and ALMA positions. The 2007 Hipparcos refined abscissa measurements required the addition of so-called Cosmic Noise of 2.4 mas to find an acceptable 5parameter stochastic solution. We find that a measure of radio Cosmic Noise should also be included for the radio positions because surface inhomogeneities exist at a level significant enough to introduce additional intensity centroid uncertainty. Combining the 2007 HIAD with the proper motions based solely on the radio positions leads to a parallax of $\pi=5.27 \pm 0.78$ mas $\left(190_{-25}^{+33} \mathrm{pc}\right)$, smaller than the Hipparcos 2007 value of $6.56 \pm 0.83$ mas $\left(152_{-17}^{+22}\right.$ pc; van Leeuwen 2007). Furthermore, combining
\end{abstract}

graham.harper@colorado.edu 
the VLA and new e-MERLIN and ALMA radio positions with the 2007 HIAD, and including radio Cosmic Noise of 2.4 mas, leads to a nominal parallax solution of $4.51 \pm 0.80$ mas $\left(222_{-34}^{+48} \mathrm{pc}\right)$, which while only $0.7 \sigma$ different from the 2008 solution of Harper et al. it is $2.6 \sigma$ different from the solution of van Leeuwen. An accurate and precise parallax for Betelgeuse is always going to be difficult to obtain because it is small compared to the stellar angular diameter $(\theta=44$ mas). We outline an observing strategy, utilizing future $\mathrm{mm}$ and sub-mm high-spatial resolution interferometry that must be used if substantial improvements in precision and accuracy of the parallax and distance are to be achieved.

Keywords: stars: astrometry — stars: individual $(\alpha$ Ori) 


\section{INTRODUCTION}

The parallax of the red supergiant Betelgeuse ( $\alpha$ Ori: M2 Iab) is of considerable interest to the astrophysics community because it is required to determine the fundamental stellar parameters, and to constrain its evolutionary status, e.g., an age of $\sim 8-10 \mathrm{Myr}$, with $<1 \mathrm{Myr}$ until it explodes (Harper et al. 2008; Dolan et al. 2016). There is a paucity of M supergiants in the solar neighborhood, and Betelgeuse is one of only two nearby supernova progenitors - the other being Antares (M1 Iab $+\mathrm{B} 3 \mathrm{~V}$ : dist $\simeq 200 \mathrm{pc}$ ). If Betelgeuse explodes as a red supergiant Betelgeuse will likely become a Type II-P, or if it evolves blueward it may become a Type II-L, see Smith et al. (2009). As a bright large angular diameter source it has been the focus of innumerable multi-wavelength observational studies, but relatively few theoretical ones. It is frustrating that its parallax is currently so poorly constrained: $5.07 \pm 1.10(1 \sigma)$ mas $\left(197_{-35}^{+55} \mathrm{pc}\right.$; Harper et al. 2008), which severely limits what can be gleaned from all the research effort into this system. The luminosity reflects the star's mass and, hence, its evolution and lifetime, and with its interferometeric angular diameter of $\simeq 44$ mas, (e.g., Ohnaka et al. 2009; Haubois et al. 2009; Montargès et al. 2016), a direct estimate of the surface gravity can be obtained. Accurate stellar parameters are also crucial to limit the parameter space of theoretical and numerical studies of stellar structure, (e.g., Freytag et al. 2002), mass loss mechanism (Hartmann \& Avrett 1984; Airapetian et al. 2000), and circumstellar structure (Harper et al. 2017).

The inherent problem for astrometric solutions for stars with angular diameters larger than their parallax is that any deviation of the center of intensity from the center-of-mass (CoM or barycenter) leads to additional systematic uncertainties. Both Betelgeuse and Antares ( $\alpha$ Sco:M1 Iab) have angular diameters, $\simeq 44$ mas, and distances near $200 \mathrm{pc}$ and their parallaxes are $\simeq 5$ mas. The release of the original Hipparcos catalog (Perryman et al. 1997; ESA 1997) suggested that Betelgeuse had a distance of $131_{-23}^{+35} \mathrm{pc}$, closer than many other astrophysical estimates, (see Harper et al. 2008, and references therein). That astrometric solution required the addition of $3.4 \pm 0.6$ mas of Cosmic Noise $^{1}$ to obtain a stochastic 5 -parameter astrometric solution [i.e., R.A. $(\alpha)$, decl. $(\delta)$, parallax $(\pi)$,

\footnotetext{
${ }^{1}$ A stochastic solution is one in which the dispersion of the abscissa residuals about the optimum solution was greater than expected and an additional source of Cosmic Noise (or Dispersion) was added to the residual uncertainties.
} 
and proper motions in $\alpha \cos \delta$ and $\delta$ ]. The specific origin of this additional positional uncertainty remains unknown: potential origins include source size, brightness, instrumental effects, and movement of the stellar photo-center which may result from large-scale convective/pulsation-induced motions in the outer layer of the star (e.g., Freytag et al. 2002). Boboltz et al. (2003) derived somewhat different proper-motions from the 1997 Hipparcos solution, and this was confirmed by subsequent multi-wavelength VLA cm-radio interferometry (Harper et al. 2008). This latter study combined VLA radio positions with the 1997 HIAD and found a greater distance $\left(197_{-35}^{+55} \mathrm{pc}\right)$ and it also revealed a tension whereby the radio positions alone suggested a greater distance.

Just at the completion of the Harper et al. (2008) study, revised Hipparcos astrometry was published by van Leeuwen (2007, hereafter referred to as the 2007 Hipparcos solution). The astrometric solutions from this impressive re-analysis are generally of significantly improved quality compared to the 1997 release, and the number of single star astrometric solutions requiring Cosmic Noise was reduced from 1561 to 588. Betelgeuse's revised Hipparcos parallax put it at $152 \pm 20 \mathrm{pc}$, but the solution still required 2.4 mas of Cosmic Noise. Note that Antares also required 3.6 mas of Cosmic Noise. Recalling that the parallax derived by Harper et al. (2008) was based on the combination of VLA radio positions and the Hipparcos Intermediate Astrometric Data (Perryman et al. 1997; ESA 1997, HIAD), we have been asked whether the revised van Leeuwen (2007) intermediate astrometric data would lead to a different VLA+Hipparcos combined solution. Here we address this question and provide updated astrometric solutions.

The importance of the radio positions for the astrometric solution is the long timeline that they provide when compared to the Hipparcos mission. While Hipparcos observed the sky for 3.2 years, Betelgeuse was observed for less than 28 months. In contrast, the radio time-line now extends from 1982 to $2016(\Delta t=34 \mathrm{yr})$, and since 2008 Betelgeuse has also been observed with e-MERLIN (Garrington \& Beswick 2016) by Richards et al. (2013) and ALMA. The yearly proper motions of Betelgeuse are $\simeq 10-25$ mas yr$^{-1}$, which is a significant fraction of the angular diameter per year, so the long timeline helps to define the proper-motion elements of the astrometric solution even with modest measurement uncertainties. 
In $\S 2$ we present the new radio positions, discuss the magnitude of expected Cosmic Noise for the radio positions, and derive radio-only proper motions. In $\S 3$ we give the combined radio and 2007 Hipparcos astrometric solutions, and in $\S 4$ we discuss what is needed to find a significantly improved parallax for Betelgeuse. Conclusions are given in $\S 5$.

\section{NEW RADIO POSITIONS}

The new radio positions, which consist of two ALMA observations at 330-345 GHz and three eMERLIN observations obtained at $5.75 \mathrm{GHz}(5.2 \mathrm{~cm})$ (Richards et al. 2013), are given in Table 1.

\subsection{ALMA}

Betelgeuse was observed by ALMA in a long baseline configuration on 2015 November 9 and again on 2016 August 16 in a more compact configuration (Project code: 2015.1.00206.S , PI: P. Kervella). Both sets of observations were carried out in Band 7 with identical spectral setups and will be described in more detail in a future publication (P. Kervella, et al. 2017, in preparation) For the purpose of this work, the channels containing line emission were excluded from the analysis and a single continuum data set centered at $\simeq 338 \mathrm{GHz}$ with a $\sim 5.9 \mathrm{GHz}$ bandwidth was used. The maximum baseline in the long baseline observation was $16 \mathrm{~km}$, which yielded an angular resolution of 15 mas, while the maximum baseline provided by the more compact configuration observation was $1.2 \mathrm{~km}$ and yielded an angular resolution of 185 mas. The observations of Betelgeuse (Star) were interleaved with observations of the compact phase calibrator J0552+0313 (PhaseCal) located approximately $4 \mathrm{deg}$ away from the target using a 2-min cycle time. The compact source J0605+0939 (ChkSource) located approximately $7 \mathrm{deg}$ from the PhaseCal was used as a check source. ChkSource was found to be located 3.3 mas away from its expected position. For the compact configuration the same phase calibrator (J0552+0313) was again used but a longer cycle time of 7.5 min was implemented. J0603+0622 was used as a check source and was found to be located 15 mas away from its expected position. For both epochs, the source position was extracted from the un-selfcalibrated continuum datasets using the Python-based task uvmultifit (Martí-Vidal et al. 2014) and fitting a uniform intensity elliptical disk to the calibrated visibilities. 
The positional uncertainties $(U n c)$ for the two Betelgeuse observations are dominated by the phasereferencing uncertainty (and not fit errors). We used the difference between the measured positions of ChkSource from those expected, $\Delta \mathrm{CS}$, scaled by the relative separation of Star and PhaseCal, and the ChkSource and PhaseCal, i.e.,

$$
U n c=\Delta \mathrm{CS} \times(\text { Star }- \text { PhaseCal }) /(\text { ChkSource }- \text { PhaseCal })
$$

These uncertainties for the ALMA positions are given in Table 2. During the compact configuration observation, the wind speed was high and increasing during the observations, which increased the atmospheric instability. This in turn might lead to an underestimation of the uncertainty reported in Table 2.

\section{2. e-MERLIN}

Betelgeuse was observed in the first semester of e-MERLIN open time in 2012 July, with a bandwidth of $512 \mathrm{MHz}$ centered on $5.75 \mathrm{GHz}(5.2 \mathrm{~cm})$. Seven antennas were used, including the $75 \mathrm{~m}$ Lovell telescope, providing baselines from 11-217 km (90-3910 k $\lambda$ ). The data were processed in dual polarization, using $4 \times 128 \mathrm{MHz}$ spectral windows, each divided into $64 \times 2 \mathrm{MHz}$ channels.

The point-like QSO 0551+0829, separation $\sim 1.5$, was used as the phase reference on a cycle of 7:3 minutes. OQ208 was used as the bandpass and flux density calibrator. The flux scale is accurate to $\sim 10 \%$. The calibrated and edited Betelgeuse data comprised 4-8hr per antenna, spread over $10.5 \mathrm{hr}$, with an average bandwidth of $400 \mathrm{MHz}$. The data were imaged using two schemes, one for highest resolution and the other, with a slight tapering of the visibilities, for maximum sensitivity to the extended stellar atmosphere, using a 180-mas circular restoring beam. The latter was used to measure the astrometric position. The astrometric uncertainty arising from phase referencing is 16 mas, and adding the noise-based fitting uncertainty gives a total of 17.5 mas. Richards et al. (2013) describes these observations in more detail, but note that the measurements given in this paper are after re-processing using the correct receiver mount position for the Cambridge antenna (Beswick 2015). 
Similar observations were made in 2015 March and June, but the phase reference 0605+0939 was used, $\sim 7^{\circ}$ from the target, giving a total astrometric uncertainty of 22.2 and 22.5 mas in March and June, respectively.

The total flux densities at successive epochs were 2.78, 2.39 and $2.35 \mathrm{mJy}$ in areas $204 \times 195$, $212 \times 198$ and $201 \times 189 \mathrm{mas}^{2}$, respectively, which is consistent with the barely significant ellipticity measured in VLA data. At all epochs, the high-resolution images (as reprocessed, for 2012) show different distributions of six to eight small hot spots at the level of $\approx 10 \%$ of the total flux density. We tested whether these were biasing the fits by subtracting the hot spots from the 2012 visibility data and replacing them with the 2015 March hot spots, and re-imaging the modified 2012 data at 180-mas resolution. The position fitted was less than 1 mas different from the original measurement, suggesting that the hot spots seen at $5.75 \mathrm{GHz}$ do not significantly bias the (tapered) measured e-MERLIN centroid position and thus are not a significant source of Cosmic Noise for this analysis.

\subsection{Sources of Additional Radio Positional Noise}

In Harper et al. (2008) the radio positions were assigned positional uncertainties under the assumption that the intensity weighted center was the position of the barycenter. However, the Lim et al. (1998) VLA Q-band $(7 \mathrm{~mm}, 43 \mathrm{GHz})$ map shows the star is not axisymmetric being better fit with a $95( \pm 2) \times 80( \pm 2)$ mas ellipse with a position angle $(\mathrm{PA})$ of $67( \pm 7)$ deg (East of North). The presence of non-axisymmetric intensity distribution directly implies that one must consider the possibility of an offset between the weighted intensity centroid and the star's barycenter. Elliptical fits have been made in subsequent multi-epoch multi-wavelength VLA maps (O'Gorman et al. 2015) and the orientation does not appear to reflect a fundamental property of the star, e.g., rotational distortion (Tatebe et al. 2007), and furthermore, the PA varies with time. For example, an elliptical fit to the ALMA high-spatial resolution map ( 330 GHz), which samples closer to the photosphere than the VLA Q-band, gives 54.50( \pm 0.01$) \times 50.44( \pm 0.01)$ mas with a PA=155.5 $( \pm 0.2)$ deg $\left(\mathrm{O}^{\prime}\right.$ Gorman et al. 2017).

The problem then is how do we quantify the magnitude of the intensity centroid shift with respect to the barycenter? At this point in time nothing rigorous can be done because reliable grids of physically 
complete 3D atmospheric models describing this part of the atmosphere do not exist. Chiavassa et al. (2011) considered optical photo-center motions from radiative hydrodynamic simulations of a red supergiant like Betelgeuse that uses only gray opacities in the visible surface layers. They find a dispersion of 0.5 mas which is consistent with ground-based interferometry obtained by Wilson et al. (1992) (0.4 mas) and Tuthill et al. (1997) (1.2 mas), but still smaller than the Hipparcos Cosmic Noise of 2.4 mas. More recently, Montargès et al. (2016) have presented H-band interferometry that reveals larger photo-center displacements at 4 epochs over 3 years with a mean displacement of 1.2 mas. Furthermore, during 2014, the photo-center moved by 3.4 mas. The H-band covers the $1.6 \mu \mathrm{m} \mathrm{H}^{-}$ opacity minimum and samples the deeper, more convective layers. SPHERE/ZIMPOL images from the VLT, with a resolution of $\sim 20$ mas, also reveal non-symmetric photospheric emission in the $\mathrm{V}(\lambda 554 \mathrm{~nm} ; \Delta \lambda=81 \mathrm{~nm})$ and TiO717 filters $(\lambda 717 \mathrm{~nm} ; \Delta \lambda=20 \mathrm{~nm})$ (Kervella et al. 2016). The global asymmetries observed with SPHERE/ZIMPOL can introduce apparent photo-center offsets of order $\sim 1$ mas.

For the high-spatial and high signal-to-noise ratio ALMA 2015 map there are residuals to the uniform intensity ellipse fits. Eight different fits to the ALMA data; an ellipse with additional Gaussian spots, rings, and point sources, lead to shifts in the apparent ellipse centroid with a standard deviation of 0.43 mas. If we take these fits as possible realizations of radio-center displacements, then we can take 0.43 mas as a measure of the minimum of additional radio noise - that might correspond to the Hipparcos Cosmic Noise. However, the ratio of major to minor axis length of 1.1 suggests the potential for larger radio-center offsets. If we take the minor axis as representative of an unperturbed star then there is a 5 mas extension for the other axis. We adopt approximately half this value as the nominal radio noise, i.e. $\sigma_{\text {radio }}^{\text {noise }}=2.4$ mas. The similarity to the Hipparcos Cosmic Noise in the 2007 solution may be coincidental because the sensitivity of specific intensity to changes in gas temperature are much smaller on the Rayleigh-Jeans tail of the Planck function. Including this additional uncertainty in the radio positional errors (in quadrature) only has an effect on the few high-spatial resolution radio observations, which, apart from one, are from the VLA. 
Simulations of optical photo-center displacement show time-scales of months and years (Chiavassa et al. 2011), so the photo-center displacements are not random, as assumed in the Hipparcos astrometric fitting procedures. However, at this point there is little more that we can do except to point out a potential future solution in $\S 6.1$.

\section{RADIO-ONLY PROPER MOTIONS AND PARALLAX}

It has already been noted that VLA radio positions suggest different proper motions to the 1997 Hipparcos astrometric solution (Boboltz et al. 2003; Harper et al. 2008) [see Table 2]. The differences become more significant now because the 2007 Hipparcos solution has uncertainties that are a factor of $>2$ smaller.

Here, we consider the new radio dataset and explore the proper motions and parallaxes that result from different levels of additional radio centroid noise. We compute the parallaxes using the JPL DE405 IRCF ephemerides (Standish 1998). These proper motions are presented in Table 3 where the uncertainties are calculated assuming the adopted astrometric model is the correct one. The parallaxes are not tightly constrained because there are only a few positions with small positional uncertainties comparable to the size of the parallax. The parallaxes are smaller than given by either the 1997 and 2007 Hipparcos solutions and the parallax increases with increasing radio Cosmic Noise. Considering Table 2 , it can be seen that none of the proper motions $\left(\mu_{\alpha \cos \delta}\right.$ or $\left.\mu_{\delta}\right)$ agree with the 2007 Hipparcos solution. This suggests that there is a bias in Hipparcos proper motions that may arise from too much weight being given to the direction $29^{\circ}$ E. of N. The PA of Betelgeuse's rotation axis, as deduced from combined UV spectral measurements, is $\sim 65^{\circ}$ E. of N. (Harper \& Brown 2006; Uitenbroek et al. 1998) and with the a period of $\geq 17 \mathrm{yr}$ equatorial motion of bright regions is unlikely to be an explanation. However, 1991-1992 interferometry from Wilson et al. (1992) and Tuthill et al. (1997) show hot spots which shift the photo-center with respect to the extended disk to PA's of $39^{\circ}$ (1991 January) and $-29^{\circ}$ (1992 January) which can be attributed to photospheric convective motions (Chiavassa et al. 2011). This provides evidence that photo-center motions did occur during the Hipparcos mission, and these may have contributed to the necessity of adopting a stochastic solution. 


\section{REVISED INTERMEDIATE ASTROMETRIC SOLUTIONS}

The HIAD were retrieved from the DVD included in van Leeuwen (2007, Appendix G2.1)2 . There are 66 scan level abscissa (a) measurements, and we reject one outlier measurement as in the 2007 published solution. This represents an increase in the number of measurements from the original reduction (38). van Leeuwen (2007, Eq. 2.52) gives the differences between predicted and measured abscissae (residuals, $\mathrm{d} a$ ) as

$$
\mathrm{d} a=\cos \psi \mathrm{d} \alpha \cos \delta+\sin \psi \mathrm{d} \delta+\operatorname{PARF} \mathrm{d} \pi+\cos \psi \Delta t \mathrm{~d} \mu_{\alpha \cos \delta}+\sin \psi \Delta t \mathrm{~d} \mu_{\delta}
$$

where $\pi$ is the parallax and PARF (known as the parallax factor) $\psi$ is related to the scan angle. For each scan, the estimated abscissa uncertainties $\sigma_{a}$ are also provided. For the stochastic solution $\sigma_{a}$ includes the additional Cosmic Noise of $\sigma_{H i p p}^{\text {noise }}=2.4$ mas. The residuals from the van Leeuwen (2007) solution, $\mathrm{d} a$, PARF, $\psi$, and $\Delta t$ are given in the DVD. The published solution represents the minimum of

$$
\chi^{2}=\sum_{i=1}^{65}\left(\frac{\mathrm{d} a}{\sigma_{a}}\right)^{2}
$$

Introducing information from the radio positions, e.g. proper motions, leads to perturbations about the 2007 Hipparcos solution, i.e., $\mathrm{d} \alpha \cos \delta, \mathrm{d} \delta, \mathrm{d} \pi, \mathrm{d} \mu_{\alpha \cos \delta}$, and $\mathrm{d} \mu_{\delta}$ whose effect on the optimum solution can then be explored using Eqs 1 and 2. Both the radio and 2007 Hipparcos solutions are given in the International Celestial Reference System (ICRS). The uncertainty in the link between the 1997 Hipparcos catalog (Perryman et al. 1997) and ICRS at J1991.25 was 25 mas yr $^{-1}$ in the relative motions of the frames, and the Hipparcos 2007 catalog is in agreement to within the uncertainties (van Leeuwen 2007, S3.6).

\section{RESULTS}

\subsection{Radio Fixed Proper Motions and HIAD}

\footnotetext{
${ }^{2}$ There is a very small difference in some of the adopted astrometric values available on-line and on the DVD.
} 
The long time span of the radio observations provides a powerful constrain on the proper motions. By fixing $\mu_{\alpha \cos \delta}$ and $\mu_{\delta}$ for the case of $\sigma_{\text {radio }}^{\text {noise }}=2.4$ mas (Table 2) in Eqs 1 and 2 we find a parallax of $\pi=5.27 \pm 0.78$ mas. This gives a distance of $190_{-25}^{+33} \mathrm{pc}$ which is larger than the Hipparcos revised 2007 value of $152_{-17}^{+22}$ pc. Examination of the correlation coefficients of the 2007 Hipparcos solution shows that the parallax is positively correlated with both proper motions. The radio proper motions are both smaller than the 2007 Hipparcos solution so the parallax is also smaller, and the distance greater.

\subsection{Radio Positions and HIAD}

New astrometric solutions were then obtained using the revised HIAD combined with the full radio positional data in Table 1 and Harper et al. (2008, and references therein). We minimized the $\chi^{2}$ from the residuals in Eqs. 1 and 2 combined with the radio position offsets $(d \alpha \cos \delta$ and $d \delta)$ weighted by their uncertainties. We used Levenberg-Marquardt least-squares minimization (Markwardt 2009) with the 65 1D HIAD scans and each of the 18 2D radio position. For the radio, the residuals in $d \alpha \cos \delta$ and $d \delta$ were evaluated separately, effectively giving 36 datum $(2 \times 18$ positions $)$, for a total of 101 individual evaluations. The result, assuming $\sigma_{\text {radio }}^{\text {noise }}=2.4$ mas, is given in Table 4 , along with the 2007 Hipparcos solution. For convenience, the position offsets for the 1991.25 Epoch are given with respect to the 2007 Hipparcos solution. Figure 1 (top) depicts this solution along with the radio positions, while the bottom panel shows the 2007 Hipparcos solution.

The most significant difference between the radio+Hipparcos and the 2007 Hipparcos solution is the proper motion in declination where the 2007 value of $11.32 \pm 0.65$ compares to $9.60 \pm 0.12 \mathrm{mas} / \mathrm{yr}$. This illustrates the importance of the long time span of the radio observations, and again hints that there is a preferred direction of the effective optical photo-center displacement during the Hipparcos observing epoch. Astrophysically, the most important result is that the combined radio+Hipparcos parallax is $4.51 \pm 0.80$ mas or $222_{-33}^{+48} \mathrm{pc}$, which is $0.7 \sigma$ greater than that found by Harper et al. (2008). Both the solution using just the radio proper motions, and the combined radio+Hipparcos lead to a smaller parallax and greater distance than either of the published Hipparcos results. Figure 2 shows the sensitivity of the new parallax to the adopted radio cosmic noise. Above 2.4 mas the few precise radio 
positions lose their impact and the combined solution is controlled by the proper-motion constraints (which remain insensitive to the adopted value of $\sigma_{\text {radio }}^{\text {noise }}$. There is certainly an argument to be made that the parallax could be smaller by adopting a smaller $\sigma_{\text {radio }}^{\text {noise }}$ in the combined solution.

The tension between the optical (closer distance) and radio (farther distance) means that any changes in the relative weighting of the two datasets, either through the number of effective measurements or the additional added Cosmic Noise, will shift the parallax to some degree. However, these results show that Hipparcos still overestimates the parallax.

We note that Betelgeuse is very bright, too bright for GAIA to observe, and it is not clear that the small Japanese Nano-Jasmine $5.25 \mathrm{~cm}$ IR telescope satellite ${ }^{3}$ will be able to get useful data for Betelgeuse (if it is launched).

\section{DISCUSSION}

While the astrometric solutions presented above are probably the best that can be done at the present time, it is not the final solution to the problem of the stellar distance. The present parallax for Betelgeuse implies it is 1.2 times more luminous than in Harper et al. (2008), but the uncertainties remain significant for astrophysical purposes. To obtain a solid parallax measurement requires multiple observations where one could simultaneously measure the intensity position, with reference to accurate position (phase) calibrators, and image the uneven intensity distribution on the stellar surface. In this way, one has the chance that the continuum centroid and uncertainties from surface intensity variations could be estimated with sufficient precision to enable a reliable parallax determination.

\subsection{What is the optimum wavelength to make astrometric measurements?}

The outer atmosphere of the convective M supergiant may be shaped by gas pressure, rotation, convective motions, shocks, and magnetic fields competing with the stellar gravitational field. These may lead to surface brightness variations that are not centered on the star's barycenter which in

${ }^{3}$ https://directory.eoportal.org/web/eoportal/satellite-missions/n/nano-jasmine 
turn will influence the astrometric solution. For Betelgeuse, rotation is not expected to lead to significant surface distortion (Tatebe et al. 2007). One would like to sample the layers well above the optically-thin visual surface and where convection-induced height variations are minimized. Surface magnetic fields are expected to decrease more slowly with height than the gas density (pressure) and magnetic fields are expected to shape the outer layers (Harper 2013), including the hotter ultraviolet emitting chromospheric region near $2 R_{*}$. When the gas temperature increase to above $\sim 4000 \mathrm{~K}$, hydrogen becomes a significant electron donor and the radio opacity can increase significantly. This suggests that an optimal radial location for astrometric measurements is well above the photosphere but interior to the higher temperature chromosphere. This region is still relatively uncharted and is expected to host low-temperature inhomogeneities related to the MOLsphere phenomenon (Tsuji 2006; Ohnaka et al. 2009), which is probably the cooler and lower-gravity equivalent of the COmosphere phenomenon observed in warmer stars, including K giants and the Sun (Wiedemann et al. 1994; Ayres 2002).

The intensity changes $(\Delta I)$ resulting from differences in temperature $(\Delta T)$ in a given layer are expected to be less in the sub-mm and $\mathrm{cm}$ than in the optical because the thermal continuum emission is on the Rayleigh-Jeans tail where perturbations should be linear in temperature, i.e., $\Delta I \propto \Delta T$. In the optical, the sensitivity is greater because of the exponential nature of the Planck function, and in the V-band there is additional sensitivity resulting from the sensitivity of molecular opacity to temperature.

Fortunately, the $\mathrm{mm}$ and sub-mm radio frequencies probe the optimum atmospheric layers, and in addition, high-spatial resolution observations can image these layers at the same time as the position is accurately known with respect to an external reference frame (ICRS). This may be achieved with ALMA in the sub-mm, and perhaps in combination with the JVLA at Q-band (43 GHz, $6.9 \mathrm{~mm})$. These observations would need maximum baseline configurations for the highest spatial resolution.

To obtain an accurate and precise parallax for Betelgeuse will require a dedicated multi-year observing plan. In addition to requiring good weather conditions for atmospheric phase stability, Betelgeuse observations with multiple check sources are needed near maximum parallax shift. Redundant ob- 
servations will need to be planned because experience shows that data will be lost to bad weather. Another challenging constraint is that interferometer array configurations are routinely changed in non-yearly cycles to satisfy other science priorities.

\section{CONCLUSIONS}

The VLA+ 1997 Hipparcos HIAD astrometric solution for Betelgeuse (Harper et al. 2008) has been improved by using the revised 2007 Hipparcos HIAD from van Leeuwen (2007) combined with extant VLA data and new e-MERLIN and ALMA positions. The non-axisymmetric intensities found in high-spatial resolution VLA and ALMA radio maps indicate that the intensity centroid may not coincide with the barycenter, leading us to introduce radio Cosmic Noise analogous to that required for the Hipparcos optical solutions.

First, we find that using the proper motions from the radio data alone, which has a time span of 34 years, leads to a smaller parallax (greater distance) than either Hipparcos solution (time span of 28 months). Second, the new combined radio+Hipparcos astrometric solution gives a parallax and distance of $4.51 \pm 0.80$ mas and $222_{-34}^{+48} \mathrm{pc}$, respectively. This distance is slightly larger, but

not significantly so, than the previous radio+Hipparcos solution of $197_{-35}^{+55}$ pc (Harper et al. 2008). However, the new solutions are significantly different from the 2007 Hipparcos solution of van Leeuwen (2007). To obtain a more precise parallax will require high-spatial resolution continuum observations in the mm and sub-mm that can be obtained with ALMA and JVLA. Scheduling and obtaining the required observations may take a Herculean effort.

Financial support for this work was provided by NASA through a SOFIA grant NAS2-97001 issued by USRA to GMH, who also acknowledges the assistance of R.W.H. P.K. acknowledges financial support from the Programme National de Physique Stellaire (PNPS) of CNRS/INSU, France. E.O.G. acknowledges support from the Irish Research Council. L.D. acknowledges support from the ERC consolidator grant 646758 AEROSOL and the FWO Research Project grant G024112N. This paper makes use of the following ALMA data: ADS/JAO.ALMA\#2015.1.00206.S. ALMA is a partnership of ESO (representing its member states), NSF (USA) and NINS (Japan), together with NRC (Canada), 
NSC and ASIAA (Taiwan), and KASI (Republic of Korea), in cooperation with the Republic of Chile. The Joint ALMA Observatory is operated by ESO, AUI/NRAO and NAOJ. The National Radio Astronomy Observatory is a facility of the National Science Foundation operated under cooperative agreement by Associated Universities, Inc. This research has made use of the VizieR catalogue access tool, CDS, Strasbourg, France. It has also made use of NASA's Astrophysics Data System Bibliographic Services. Finally, we thank the referee for their insights.

Facilities:

Facility: Hipparcos,

Facility: VLA,

Facility: ALMA,

Facility: e-MERLIN. 

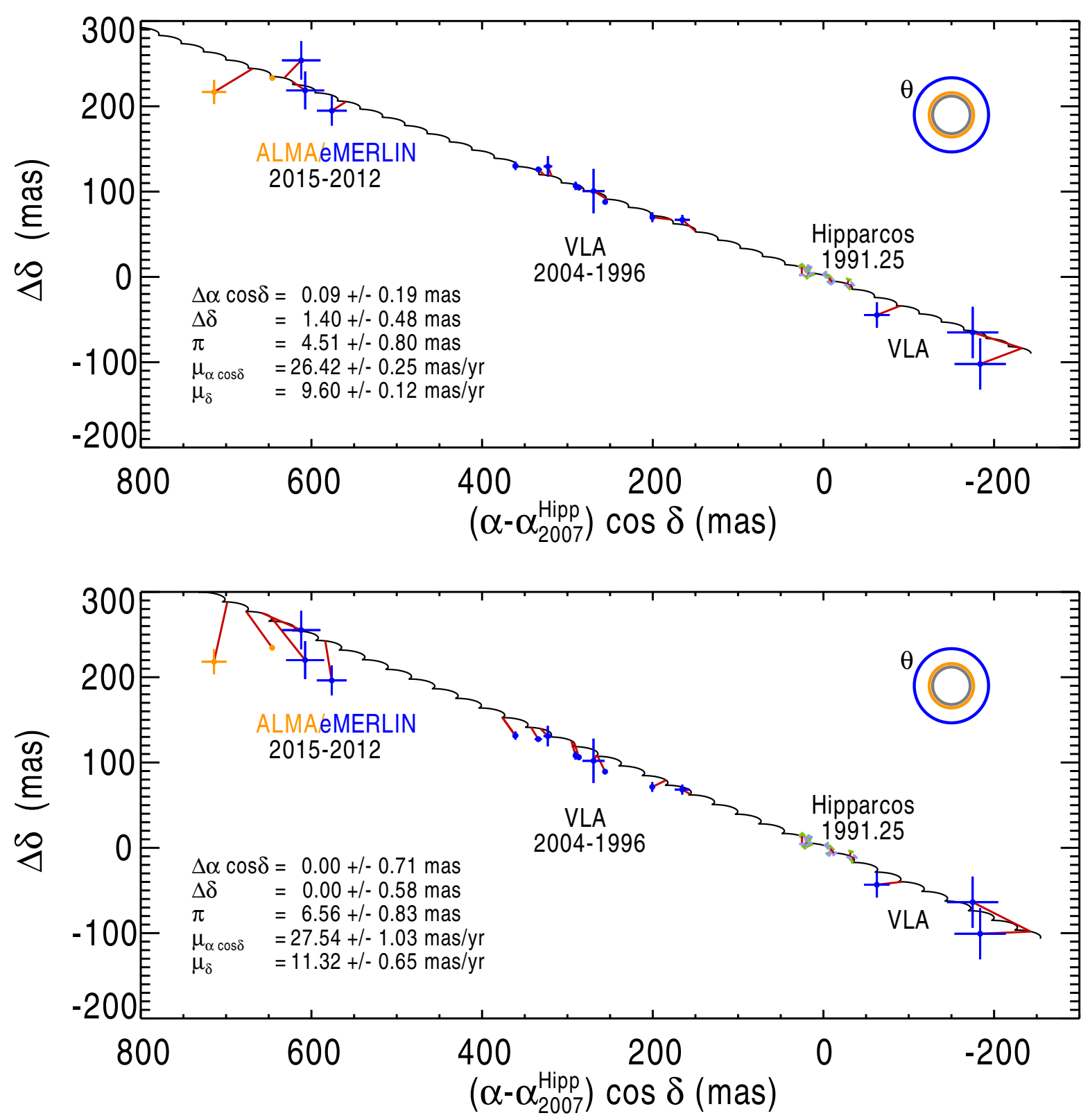

Figure 1. Top: The new astrometric solution computed with an assumed radio Cosmic Noise of 2.4 mas. The 1997 HIAD Hipparcos data are shown for illustration (see Harper et al. 2008). On this scale the 1997 Hipparcos data are not distinguishable from the 2007 data. The apparent angular diameter of the star at different wavelengths is shown in the upper-right corner for reference - inner circle is H-band $(1.6 \mu \mathrm{m})$, the yellow is ALMA $(338 \mathrm{GHz})$ and blue is Q-band $(43 \mathrm{GHz})$. Bottom: The 2007 Hipparcos solution which shows how the radio data constrains the proper-motions. The radio data show that Hipparcos systematically overestimates $\mu_{\delta}$ and to a lesser extent $\mu_{\alpha \cos \delta}$. 


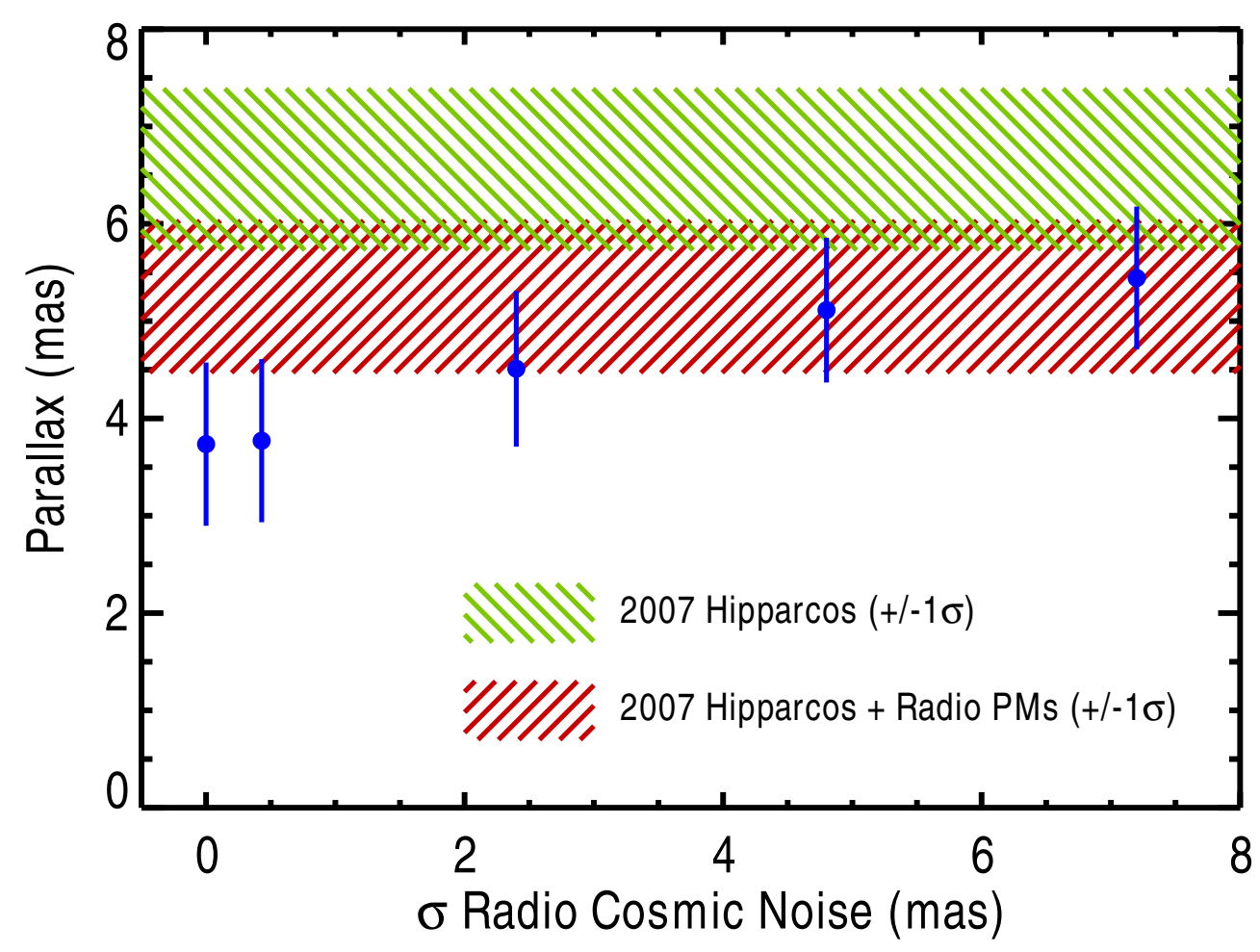

Figure 2. The parallax from the combined radio and 2007 Hipparcos HIAD data as a function of Radio Cosmic Noise $\left(\sigma_{\text {radio }}^{\text {noise }}\right)$. The top hashed area (green) is the 2007 Hipparcos solution, and the hashed area below (red) is Hipparcos HIAD combined with the radio-only proper motions (this work, assuming $\sigma_{\text {radio }}^{\text {noise }}=$ 2.4 mas). The combined radio+Hipparcos solutions are shown as circular symbols and error bars (blue) for different values of assumed $\sigma_{\text {radio }}^{\text {noise }}$. When $\sigma_{\text {radio }}^{\text {noise }}>3$ mas, the sensitivity of the parallax to the radio positions is reduced. 
Table 1. New ICRS Radio Positions for Betelgeuse.

\begin{tabular}{lcccc}
\hline Date & RA & Decl. & Unc. & Reference \\
& $(\mathrm{h} \mathrm{m} \mathrm{s})$ & $\left({ }^{\circ} \quad \prime^{\prime \prime}\right)$ & $($ mas $)$ & \\
\hline 2012.530 & 055510.32672 & 072425.5263 & 17.5 & $5.75 \mathrm{GHz}$, Richards et al. (2013) \\
2015.178 & 055510.32882 & 072425.5501 & 22.2 & $5.75 \mathrm{GHz}$, New \\
2015.422 & 055510.32913 & 072425.5853 & 22.5 & $5.75 \mathrm{GHz}, \mathrm{New}$ \\
2015.854 & 055510.33142 & 072425.5646 & 2.0 & $330-345 \mathrm{GHz}$ ALMA \\
2016.623 & 055510.33560 & 072425.5482 & 14.1 & $330-345 \mathrm{GHz}$ ALMA \\
\hline
\end{tabular}

Table 2. Proper Motions for Betelgeuse ${ }^{\mathrm{a}}$.

\begin{tabular}{lcccc}
\hline Reference & $\mu_{\alpha \cos \delta}$ & $\sigma_{\mu_{\alpha \cos \delta}}$ & $\mu_{\delta}$ & $\sigma_{\mu_{\delta}}$ \\
\hline ESA (1997) & 27.33 & 2.30 & 10.86 & 1.46 \\
Boboltz et al. (2007) & 23.98 & 1.04 & 10.07 & 1.15 \\
Harper et al. (2008) & 24.95 & 0.08 & 9.56 & 0.15 \\
van Leeuwen $(2007)$ & 27.54 & 1.03 & 11.30 & 0.65 \\
Radio-only [ $\sigma_{\text {radio }}^{\text {noise }}=2.4$ mas] & 25.53 & 0.31 & 9.37 & 0.28 \\
\hline & $a_{\text {Units: }}$ mas yr & & &
\end{tabular}

\section{REFERENCES}

Airapetian, V. S., Ofman, L., Robinson, R. D.,

Carpenter, K., \& Davila, J. 2000, ApJ, 528, 965

Ayres, T. R. 2002, ApJ, 575, 1104

Beswick, R. 2015, e-MERLIN Technical Memo

Boboltz, D. A., Fey, A. L., Johnston, K. J., et al. 2003, AJ, 126, 484

Chiavassa, A., Pasquato, E., Jorissen, A., et al. 2011, A\&A, 528, A120
Dolan, M. M., Mathews, G. J., Lam, D. D., et al. 2016, ApJ, 819, 7

ESA, ed. 1997, ESA Special Publication, Vol. 1200, The HIPPARCOS and TYCHO catalogues. Astrometric and photometric star catalogues derived from the ESA HIPPARCOS Space Astrometry Mission 
Table 3. Radio-only Astrometric Solutions ${ }^{\mathrm{a}}$

\begin{tabular}{ccccccc}
\hline $\begin{array}{c}\text { noise } \\
\text { radio }\end{array}$ & $\pi$ & $\sigma_{\pi}$ & $\mu_{\alpha \cos \delta}$ & $\sigma_{\mu_{\alpha \cos \delta}}$ & $\mu_{\delta}$ & $\sigma_{\mu_{\delta}}$ \\
\hline 0.00 mas & 3.33 & 1.93 & 25.77 & 0.28 & 9.55 & 0.24 \\
0.43 mas & 3.38 & 1.94 & 25.75 & 0.28 & 9.54 & 0.24 \\
2.40 mas & 3.77 & 2.20 & 25.53 & 0.31 & 9.37 & 0.28 \\
4.80 mas & 4.35 & 2.67 & 25.42 & 0.35 & 9.28 & 0.32 \\
7.20 mas & 5.16 & 3.11 & 25.37 & 0.37 & 9.26 & 0.35 \\
\hline
\end{tabular}

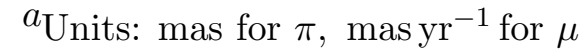

Table 4. Astrometric Solutions for Betelgeuse.

\begin{tabular}{lcc}
\hline Parameter & van Leeuwen $(2007)$ & Present Work \\
\hline$\Delta \alpha \cos \delta(\mathrm{deg})$ & $0.00(0.71)$ & $0.09(0.19)$ \\
$\Delta \delta(\mathrm{deg})$ & $0.00(0.58)$ & $1.40(0.48)$ \\
Parallax $\pi(\mathrm{mas})$ & $6.55(0.83)$ & $4.51(0.80)$ \\
$\mu_{\alpha \cos \delta\left(\operatorname{mas~yr}^{-1}\right)}$ & $27.54(1.03)$ & $26.42(0.25)$ \\
$\mu_{\delta}\left(\operatorname{mas~yr}^{-1}\right)$ & $11.30(0.65)$ & $9.60(0.12)$ \\
\hline
\end{tabular}

Freytag, B., Steffen, M., \& Dorch, B. 2002,

Astronomische Nachrichten, 323, 213

Garrington, S., \& Beswick, R. 2016, Astronomy and Geophysics, 57, 3.28

Harper, G. M. 2013, in EAS Publications Series, Vol. 60, EAS Publications Series, ed.

P. Kervella, T. Le Bertre, \& G. Perrin, 59-68

Harper, G. M., \& Brown, A. 2006, ApJ, 646, 1179

Harper, G. M., Brown, A., \& Guinan, E. F. 2008, AJ, 135, 1430
Harper, G. M., DeWitt, C., Richter, M. J., et al. 2017, ApJ, 836, 22

Hartmann, L., \& Avrett, E. H. 1984, ApJ, 284, 238

Haubois, X., Perrin, G., Lacour, S., et al. 2009, A\&A, 508, 923

Kervella, P., Lagadec, E., Montargès, M., et al. 2016, A\&A, 585, A28

Lim, J., Carilli, C. L., White, S. M., Beasley, A. J., \& Marson, R. G. 1998, Nature, 392, 575 
Markwardt, C. B. 2009, in Astronomical Society of the Pacific Conference Series, Vol. 411, Astronomical Data Analysis Software and Systems XVIII, ed. D. A. Bohlender, D. Durand, \& P. Dowler, 251

Martí-Vidal, I., Vlemmings, W. H. T., Muller, S., \& Casey, S. 2014, A\&A, 563, A136

Montargès, M., Kervella, P., Perrin, G., et al. 2016, A\&A, 588, A130

O'Gorman, E., Harper, G. M., Brown, A., et al. 2015, A\&A, 580, A101

O'Gorman, E., Kervella, P., Harper, G. M., et al. 2017, A\&A

Ohnaka, K., Hofmann, K.-H., Benisty, M., et al. 2009, A\&A, 503, 183

Perryman, M. A. C., Lindegren, L., Kovalevsky, J., et al. 1997, A\&A, 323, L49

Richards, A. M. S., Davis, R. J., Decin, L., et al. 2013, MNRAS, 432, 61
Smith, N., Hinkle, K. H., \& Ryde, N. 2009, AJ, 137,3558

Standish, E. M. 1998, JPL IOM, 312, F

Tatebe, K., Chandler, A. A., Wishnow, E. H., Hale, D. D. S., \& Townes, C. H. 2007, ApJL, 670, L21

Tsuji, T. 2006, ApJ, 645, 1448

Tuthill, P. G., Haniff, C. A., \& Baldwin, J. E. 1997, MNRAS, 285, 529

Uitenbroek, H., Dupree, A. K., \& Gilliland, R. L. 1998, AJ, 116, 2501

van Leeuwen, F., ed. 2007, Astrophysics and Space Science Library, Vol. 350, Hipparcos, the New Reduction of the Raw Data

Wiedemann, G., Ayres, T. R., Jennings, D. E., \& Saar, S. H. 1994, ApJ, 423, 806

Wilson, R. W., Baldwin, J. E., Buscher, D. F., \& Warner, P. J. 1992, MNRAS, 257, 369 